Check for updates

Cite this: Environ. Sci.: Processes Impacts, 2020, 22, 1233

Received 20th December 2019 Accepted 24th March 2020

DOI: 10.1039/c9em00595a

rsc.li/espi

\section{Comparing non-targeted chemical persistence assessed using an unspiked OECD 309 test to field measurements $\dagger$}

\begin{abstract}
Zhe Li ${ }^{*}$ * and Michael S. McLachlan (iD
Previous research has shown that unspiked OECD 309 tests can be used to quantify chemical biodegradation in surface waters, relying on chemical residues already present in the water. Here we test the hypothesis that unspiked OECD 309 tests can quantitatively predict chemical persistence in the environment by comparing chemical half-lives assessed in the laboratory against those measured in the field. The study object was a Swedish lake heavily impacted by treated municipal wastewater. Half-lives in the field were measured by mass balance over 12 weeks. In parallel, half-lives in the lab were determined with an unspiked OECD 309 test run for 60 days. Chemical analysis was conducted using a non-target screening approach. The field study yielded a half-life $<100$ days for 38 chemicals for which the dominant source was wastewater; 32 of these were also detected in the lab test, whereby 18 had half-lives with a well-constrained uncertainty that did not intersect infinity. For 14 of the 18 chemicals, the field and lab half-lives agreed within a factor 3. In summary, the lab test predicted chemical attenuation in the field well. Limitations of the approach include the need for measurable chemical concentrations in the water body and failure to account for some attenuation mechanisms like phototransformation.
\end{abstract}

\section{Environmental significance}

Biodegradation is a significant removal pathway for many environmental organic contaminants and therefore a major determinant of chemical persistence. Previously it was demonstrated that chemical degradation kinetics can be measured using a combined unspiked OECD 309 test and non-target screening approach, and that such a test circumvents the artifacts in the standard OECD 309 test caused by adaptation of the microbial population to the chemicals spiked. The current work goes on to show that degradation kinetics measured with the unspiked test agree well with degradation kinetics measured in the field, based on a case study in a Swedish lake. This further supports the hypothesis that unspiked bottle tests can provide environmentally relevant estimates of biodegradation half-lives.

\section{Introduction}

Biodegradation is an important removal pathway for many environmental organic contaminants. It can thus be a major

\footnotetext{
Department of Environmental Science (ACES), Stockholm University, S-10691 Stockholm, Sweden. E-mail: zhe.li@aces.su.se; Tel: +4686747188

$\dagger$ Electronic supplementary information (ESI) available: Map of study site and sampling locations; non-target screening workflow parameters in Compound Discoverer; discharge rates of Sundet WWTP effluent and flow rates of Norra Bergundasjön during May 29 to August 20, 2017; meteorological data (air temperature, precipitation, and global radiation); matrix effect correction calculation; matrix effect temporal trend during the sampling period; mass flow of identified chemicals from Södra Bergundasjön (inflow), Sundet WWTP (effluent), and Norra Bergundasjön (outflow), and the percentage contribution of the inflowing water to the total chemical flow into the lake; half-lives of the detected chemicals in the lab and the field; degradation kinetics of the 14 chemicals for which the uncertainty in half-life was very large or the calculated half-life confidence intervals intersected infinity. The peak areas measured in the field study are provided in a separate file. See DOI: 10.1039/c9em00595a
}

determinant of both chemical persistence and chemical exposure. Guidelines for chemical hazard assessment in many regional and global regulations, such as the European chemicals regulation REACH, set thresholds for persistence in environmental compartments (e.g., degradation half-life in water) that need to be quantitatively evaluated. ${ }^{1}$ To assess chemical exposure, quantitative estimates of degradation halflives are also required for environmental media that influence the exposure of target organisms. ${ }^{2,3}$ Field measurements can generate environmentally relevant degradation half-lives that could fulfill both of these needs. However, field measurements of degradation half-lives are difficult and expensive to perform, and consequently rare. Also, degradation in the environment varies in space and time, so that several field measurements may be necessary to obtain a representative measure. For these reasons, standardized laboratory tests have been developed to measure chemical degradation under controlled conditions. ${ }^{4}$ 
Standardized laboratory tests to measure biodegradation have largely been developed for assessing persistence. Test conditions are standardized and often differ from conditions commonly encountered in the environment. Simulation tests that better capture the complexity of the natural environment are frequently more complicated to run, and this has led to tiered testing approaches in which more complex and realistic tests are only employed after more simple tests have indicated that the degradation process is relevant for persistence or exposure assessment of the chemical of interest. An underlying assumption in this testing approach is that higher tiered tests produce degradation rates that are relevant for the field.

A higher tiered test for aerobic biodegradation of chemicals in surface waters is the OECD 309 guideline. $^{5}$ In this test chemical attenuation is measured in aerobic natural waters spiked with test chemicals and incubated in open bottles in the laboratory. Important factors contributing to the environmental relevance of this test are the use of natural water and the possibility to incubate at field temperature. However, there are still clear weaknesses associated with the test, with one being that the test chemicals are spiked into the system, often dissolved in an organic solvent. ${ }^{6}$ In a comparison of spiked and unspiked OECD 309 test bottles using the same surface water, we showed that spiking natural aquatic systems with test chemicals can strongly influence biodegradation measurements. This effect is most likely due to the adaptation of microbial organisms. ${ }^{7,8}$ The same study demonstrated that it is possible to measure degradation kinetics in the OECD 309 test without spiking, relying instead on the chemical residues present in the surface water studied.

The current study was designed to further evaluate the environmental relevance of the unspiked OECD 309 test by comparing it with biodegradation rates measured directly in the field using a traditional mass balance approach. To this end, we selected a previously characterized Swedish lake that is the recipient for a wastewater treatment plant (WWTP) and has a water residence time of several months. For measuring chemical persistence in the field, the WWTP effluent discharged to the lake as well as surface water samples of the lake inflow and outflow were collected over a period of 12 weeks. For assessing chemical biodegradation in the laboratory, an unspiked OECD 309 test was carried out in duplicate using lake water and WWTP effluent collected at the end of the field sampling period. The data were processed using a non-target approach. Biodegradation half-lives of organic contaminants detected both in the lab test and in the field were calculated and contrasted.

\section{Materials and methods}

\subsection{Chemicals and reagents}

All chemicals (purity > 98\%) were purchased from SigmaAldrich (Steinheim, Germany) or Toronto Research Chemicals Inc. (North York, Canada) and were stored under recommended conditions until use. D- or ${ }^{13} \mathrm{C}$-labeled chemicals were purchased from Toronto Research Chemicals Inc. and CDN Isotopes (Pointe-Claire Quebec, Canada). LC/MS-grade formic acid was purchased from Sigma-Aldrich. LC/MS-grade acetonitrile and methanol were purchased from VWR (Stockholm, Sweden). Milli-Q water was produced by using a Milli-Q Integral Water Purification System (Merck Millipore, Stockholm, Sweden). All standard stock solutions were prepared in methanol and stored in the dark at $-20{ }^{\circ} \mathrm{C}$.

\subsection{Field measurements}

The study site (Fig. S1†), a lake called Norra Bergundasjön in southern Sweden, has been relatively well characterized previously by Zou et al. (2015a) and has been shown to provide good conditions for contaminant mass balance studies and have relatively homogenous contaminant levels. ${ }^{9} \quad$ Norra Bergundasjön has an average depth of $3.3 \mathrm{~m}$, a surface area of $2.2 \mathrm{~km}^{2}$, receives inflow from Lake Södra Bergundasjön and effluent from the local municipal WWTP, Sundet WWTP. The only significant outflow is via a stream on the western shore. The ratio of wastewater discharge to freshwater inflow during our study ranged from $1: 1$ to $1: 3$ (Fig. S2†). As this lake is continuously exposed to treated WWTP effluent, it likely is well adapted to contaminants present in the effluent.

Water was collected from the effluent channel of the Sundet WWTP (hereafter called effluent), from Södra Bergundasjön close to the inlet to Norra Bergundasjön (inflow), and from the stream draining Norra Bergundasjön (outflow) (Fig. S1†). For inflow and outflow, $500 \mathrm{~mL}$ grab samples were collected once per week for 12 consecutive weeks during the period of May 29 to August 20, 2017. Effluent water was collected using a permanently installed flow-proportional sampling system at the WWTP. The sampling system cooled the effluent to $2{ }^{\circ} \mathrm{C}$, and every $24 \mathrm{~h}$ effluent was transferred from the sampler to an HDPE container, which was stored frozen. After 7 days, aliquots of each daily sample were combined to give a $500 \mathrm{~mL}$ weekly flowproportional sample. All samples were stored frozen in HDPE bottles until analysis, including during transportation to Stockholm University.

\subsection{Unspiked OECD 309 test}

Lake water from Norra Bergundasjön and inflowing water from Södra Bergundasjön were collected from $10 \mathrm{~cm}$ below the surface at the end of the field sampling period on August 23, 2017. The temperature of the lake water at the time of sampling was $17^{\circ} \mathrm{C}$. The solids content was $32 \mathrm{mg} \mathrm{L}^{-1}$, a reflection of the large algae population supported by the hypertrophic conditions. Fresh effluent was collected on the same day from the WWTP. The water was stored in HDPE bottles with ample head space at $5{ }^{\circ} \mathrm{C}$ in the dark until the experiments started.

Biodegradation experiments were conducted using two test systems: natural lake water and mixture lake water that was prepared by mixing inflowing lake water with the WWTP effluent discharging to the lake $(80: 20, \mathrm{v} / \mathrm{v})$. The mixture lake water was used to increase the likelihood of detecting a substantial number of organic contaminants, with the potential disadvantage that it contained a different microbial community. Duplicated incubations were conducted, each consisting of $400 \mathrm{~mL}$ of water in a $500 \mathrm{~mL}$ glass bottle. The 
experiments were started within one day of sampling the water. Incubation conditions followed the standard OECD 309 protocol, lasting for a period of 60 days at $20 \pm 1{ }^{\circ} \mathrm{C}$ in the dark. All bottles were loosely covered with cotton wool to minimize the risk of contamination while maintaining an aerobic environment in the water. Magnetic stirrers were used to maintain well-mixed conditions. Water samples $(4 \mathrm{~mL})$ were collected in triplicate from each bottle at 11 time points (on day 0, 1, 2, 4, 7, $10,15,21,28,42$, and 60), frozen immediately, and stored at $-20{ }^{\circ} \mathrm{C}$ until analysis. Dissolved oxygen was measured at each time point and always exceeded $91 \%$.

\subsection{Analysis}

Prior to analysis, an aliquot of $1 \mathrm{~mL}$ was taken from each sample. A mixture of 32 isotope-labeled standards (Table S1†) was added to each sample before it was vortexed and filtered directly into a glass LC-vial using a $0.2 \mu \mathrm{m}$ PTFE syringe filter. All samples were analyzed with ultrahigh performance liquid chromatography coupled to a Q Exactive ${ }^{\mathrm{TM}}$ HF Hybrid Quadrupole-Orbitrap ${ }^{\mathrm{TM}}$ mass spectrometer (UHPLC-Orbitrap-MS/ MS, Thermo Fisher Scientific, San Jose, USA) using the detection of a compound. The value was chosen based on the previously explored non-target limit of detection of more than 40 chemicals.

\subsection{Calculation}

Peak areas were used for all calculations. The dissipation halflife, $t_{1 / 2}\left(\mathrm{~d}^{-1}\right)$, of a chemical in the lab test was determined by fitting first-order dissipation kinetics to the peak areas measured over the incubation period. All measurements below the cutoff threshold were removed. Regressions were performed independently for each incubation bottle using the natural logarithm of peak areas normalized to the starting peak area. Regressions were rejected when there were fewer than three data points above the detection limit. Analysis of covariance (ANCOVA) was performed at a 95\% confidence level for each chemical to test for differences between replicated bottles or between natural lake water and mixture lake water tests.

The chemical half-life in the lake was calculated from the first-order rate constant for transformation of the test chemical in the lake, $k\left(\mathrm{~d}^{-1}\right)$, which was determined by mass balance ${ }^{9}$ using the equation:

$$
k=\frac{\sum\left(A_{\mathrm{In}} \times G_{\mathrm{In}}\right)+\sum\left(A_{\mathrm{Eff}} \times G_{\mathrm{Eff}}\right)-\sum\left(A_{\mathrm{W}} \times G_{\mathrm{W}}\right)+V_{\mathrm{W}} \times\left(A_{\mathrm{W}, t_{\text {end }}}-A_{\mathrm{W}, t_{\mathrm{start}}}\right)}{\overline{A_{\mathrm{W}}} \times V_{\mathrm{W}} \times\left(t_{\mathrm{end}}-t_{\mathrm{start}}\right)}
$$

electrospray ionization (ESI). All samples were injected twice, once each under ESI positive mode and ESI negative mode. The injection volume was $100 \mu \mathrm{L}$. Separation was achieved on a Hypersil GOLD ${ }^{\mathrm{TM}}$ a Q C18 polar-endcapped column $(2.1 \mathrm{~mm}$ $\times 100 \mathrm{~mm}$; particle size of $1.9 \mu \mathrm{m}$; Thermo Fisher Scientific, San Jose, USA) with a binary mobile phase gradient consisting of (A) water and (B) acetonitrile, both containing $0.1 \%$ formic acid. The gradient was linearly ramped from $95 \%$ A to $95 \%$ B over $10.0 \mathrm{~min}$, maintained for $2.0 \mathrm{~min}$, then linearly ramped back to 95\% A within $0.1 \mathrm{~min}$ and maintained for another $1.9 \mathrm{~min}$. Throughout the whole separation, the flow rate was 0.4 $\mathrm{mL} \min ^{-1}$, the sampler compartment temperature was $10^{\circ} \mathrm{C}$, and the column temperature was $40{ }^{\circ} \mathrm{C}$.

Mass accuracy of the high-resolution Orbitrap-MS/MS was calibrated regularly in both ESI positive and negative modes. Full MS was operated with a resolution of 120000 with a scan range of 80 to $1000 \mathrm{~m} / \mathrm{z}$. Data-dependent MS/MS (dd-MS ${ }^{2}$ ) was operated with a resolution of 15000 ; the top 5 precursor ions were selected for acquiring the MS/MS profile spectrum.

\subsection{Non-target data processing workflow}

All high-resolution MS data were processed using Compound Discoverer 3.0 following the workflow from Li et al. (2018), ${ }^{\mathbf{1 0}}$ consisting of peak picking, retention time alignment, unknown detection, isotope/adduct peak grouping, compound grouping, blank subtraction, and library searching using the online database mzCloud. Relevant parameters are provided in the ESI. $\dagger$ A peak area of 1000 was used as the cutoff threshold for where $A_{\text {In }}, A_{\mathrm{Eff}}$, and $A_{\mathrm{W}}$ are the peak areas of the chemical detected in inflow water, WWTP effluent, and outflow water, respectively (surrogates for concentration, $\mathrm{mol} \mathrm{m}^{-3}$ ); $G_{\mathrm{In}}, G_{\mathrm{Eff}}$, and $G_{\mathrm{W}}$ are the volume of the inflowing water, the effluent from the WWTP, and the water leaving the lake for each week, respectively $\left(\mathrm{m}^{3}\right)$; $\sum$ indicates summation of the terms for each week over the 12 week sampling period; $t_{\text {start }}$ and $t_{\text {end }}$ are the time point of the start and end of the 12 week sampling period (d); and $V_{\mathrm{W}}$ is the volume of water in the lake $\left(\mathrm{m}^{3}\right)$, which is assumed to be constant. More details of the assumptions underlying the model are provided in the ESI. $\dagger$ For some chemicals there was a pronounced decreasing trend in $A_{\mathrm{W}}$, primarily during the early part of the sampling period. In these cases, the mass balance was conducted only during the last 6 weeks of the sampling period to obtain an estimate of $t_{1 / 2}$ that was more comparable with the laboratory test. This mass balance model is applicable when only water advection and degradation are relevant removal processes for the contaminant. To ensure that this condition was fulfilled, physical chemical properties were scrutinized for the identified chemicals and only those chemicals were retained for which $\log K_{\mathrm{AW}}$ $<0$ (this excluded chemicals for which volatilization could be a relevant removal process) and $\log D_{\text {Ow }}<5$ (this excluded chemicals for which sediment burial could be a relevant removal process). Uncertainty in the half-life was assessed via error propagation using the variance in the concentrations measured in effluent, inflowing water, and outflowing water. This will overestimate uncertainty in some cases since it 
assumes that all variability in $A_{\mathrm{W}}$ is random when assessing uncertainty in the $\left(A_{\mathrm{W}, t_{\text {end }}}-A_{\mathrm{W}, t_{\text {start }}}\right)$ term. This uncertainty estimate does not include error arising from the model assumptions.

Peak areas were used as surrogates for concentrations for all calculations since the determination of $t_{1 / 2}$ was based on relative concentration and not absolute concentration. The relationship between peak area and concentration can be influenced by matrix effects. The influence of matrix effect on the $t_{1 / 2}$ calculated from the lab test was expected to be neglectable due to the fact that matrix was anticipated to remain the same in each bottle throughout the incubation period. For field measurements, matrix effects were evaluated by using the mixture of 32 isotope-labeled standards (Table S1 $\dagger$ ) that was spiked in all the effluent, lake inflow, and lake outflow water samples. The matrix effect was then assessed for each standard by calculating the ratio of the standard's peak response between outflow and effluent, and between outflow and inflow.

\section{Results and discussion}

\subsection{Non-target screening workflow performance evaluation}

The validity and reliability of the non-target screening workflow has been demonstrated previously in a study where all spiked standards (42 native and 40 isotope-labeled organic contaminants) were unambiguously identified, supported by the retention time and MS/MS spectra matching with the corresponding reference standards. ${ }^{10}$ The performance of the workflow within the context of the present study was further evaluated by testing for the identification of 12 pharmaceuticals (acesulfame, atenolol, caffeine, carbamazepine, gabapentin, hydrochlorothiazide, metoprolol, oxazepam, paracetamol, tramadol, valsartan, and venlafaxine) used for separate spiked bottle tests conducted in parallel. ${ }^{6}$ The false negative identification number was zero; all 12 chemicals were identified in both of the duplicated bottles. The dissipation kinetics calculated using the peak areas also agreed well with the dissipation kinetics obtained from concentrations determined using targeted analytical approaches. Given the high identification confidence, the nontarget screening workflow is considered applicable and adequate for the purposes of this study.

\subsection{Matrix effects}

Matrix effects have no influence on $t_{1 / 2}$ as long as they are the same in all matrices, but they may have an impact if they are different across matrices. Therefore, we explored how matrix effects would influence the calculation of $t_{1 / 2}$ from the field measurements. Matrix effects were evaluated by comparing the peak areas of the internal standards, which were present at the same concentration in all samples analyzed. A previous study has shown that our analytical method gives similar matrix effects between WWTP influent and WWTP effluent for a large number of organic contaminants, i.e. the influent: effluent peak area ratios were close to $1 .{ }^{10}$ As shown in Fig. 1, the median outflow: inflow peak area ratios were $1.0 \pm 0.10$ for all the selected isotope-labeled standards except for glimepiride- $d_{5}$ (1.2), indicating that there was also no significant difference in the matrix effects for a wide range of analytes between Södra Bergundasjön (inflow) and Norra Bergundasjön (outflow) lake water. However, the outflow : effluent ratios differed markedly from 1.0, ranging from 1.1 for bicalutamide- $d_{4}$ to 1.8 for ketoprofen- ${ }^{13} \mathrm{C}-d_{3}$. This indicates that ionization is generally suppressed in the effluent matrix compared to lake water. As a consequence, a matrix effect correction was applied. For those analytes for which there was an isotope-labeled standard the median outflow : effluent ratio for the isotope-labeled standard was used. For the analytes for which there was no isotopelabeled standard the median outflow : effluent ratio for all of the isotope-labeled standards was used and error propagation was performed to account for the influence of the matrix effect correction on the uncertainty in $t_{1 / 2}$ (see Table S1†).

\subsection{Chemicals identified in field measurements}

In the field experiment, 90 chemicals were identified in surface water using the online database mzCloud with a matching score of $>75$ and a peak area above the threshold of 1000 in either inflow or outflow. Of these, Level I identification confidence was achieved for 22 chemicals using reference standards and Level II was achieved for the remaining $68 .{ }^{11}$ The substances included natural products, pharmaceuticals, personal care products, and industrial chemicals (see Table S2 in the ESI†). Most of them have been previously reported in Swedish surface waters. ${ }^{12-17}$ Good agreement was found between this study and Zou et al. (2015a), who studied wastewater contaminants in the same lake. Of the 12 chemicals in that study, seven were unequivocally identified here (acesulfame, carbamazepine, climbazole, fluconazole, furosemide, hydrochlorothiazide, and sulfamethoxazole). ${ }^{9}$ This is particularly notable given that Zou et al. employed a highly selective targeted analytical approach where lake water was pre-concentrated by a factor of 500 , while a direct injection approach was employed in this study.

\subsection{Chemical persistence in the field}

In applying eqn (1), the water flows were taken from a hydraulic model that utilizes the natural water flows modeled by Swedish Meteorological and Hydrological Institute (SMHI), measured effluent discharge from the WWTP, and water transfers within the watershed. Ten chemicals showed a marked decrease in concentration in Norra Bergundasjön, primarily during the early part of the sampling period, and two of these (acesulfame and amphetamine) showed a decreasing time trend in effluent. Otherwise, no pronounced time trends in concentrations were observed (the peak areas for $A_{\mathrm{In}}, A_{\mathrm{Eff}}$, and $A_{\mathrm{W}}$ for each week are provided as a separate excel file in the ESI $\dagger$ ).

Out of the 90 chemicals identified in surface water, 44 chemicals had a quantified half-life of $<100 \mathrm{~d}$. One chemical, sedanolide, was discarded due to its unreliable detection in lake water samples. All of the remaining 43 chemicals (see Table 1) fulfilled the physical-chemical property criteria for application of eqn (1). The half-lives of the 43 chemicals ranged from $<10$ d for 11 chemicals (e.g., atenolol, furosemide, 

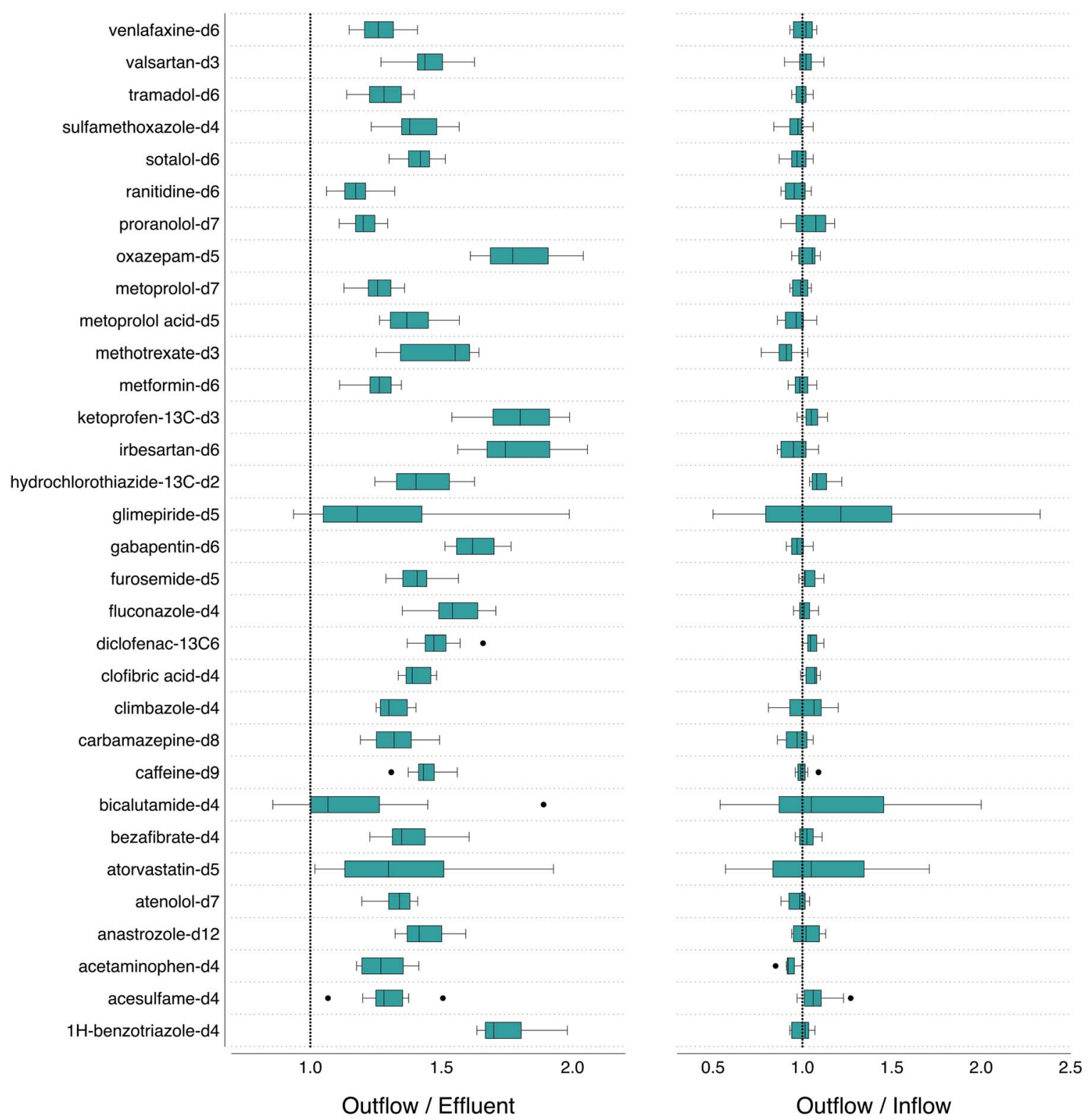

Fig. 1 Matrix effects assessed as peak area ratios of isotope-labeled standards that were present in all samples of the 3 matrices (inflow, outflow, and effluent) at equal concentrations. The medians of the 12 sample pairs are marked by a vertical line inside the boxes which span the interquartile ranges. The whiskers indicate the highest and lowest observed ratios; the dots indicate outliers.

hydrochlorothiazide, and irbesartan) to $90 \mathrm{~d}$ for choline. Correlation analysis was performed between the physicalchemical properties of the chemicals $\left(\mathrm{p} K_{\mathrm{a}}\right.$ and $\log D_{\mathrm{OW}}$ as shown in Table 1) and the calculated half-lives, yet no significant correlations were found.

For the 43 chemicals in Table 1 , the inflow from Södra Bergundasjön contributed $<30 \%$ of total inputs for 38 chemicals $(<5 \%$ for 27 ) (see Table S3 in the ESI $\dagger$ ). Therefore, the chemical inflow from Södra Bergundasjön was generally negligible compared to the chemical input from the WWTP. This was anticipated, as there are no known significant municipal wastewater discharges to the watershed upstream of Norra Bergundasjön, whereas the WWTP effluent load directly to Norra Bergundasjön is high. These findings also agree with Zou et al. (2015a). Several of the chemicals with an inflow contribution from Södra Bergundasjön of $>30 \%$ were natural products (see Table S3 $\dagger$ ). There was good agreement between halflives for the three chemicals in Table 1 that were also reported in Zou et al. (2015a): furosemide (5.9 d vs. $<9 \mathrm{~d})$, hydrochlorothiazide ( $<3.5 \mathrm{~d} v s .3 \mathrm{~d}$ ), and climbazole ( $<24 \mathrm{~d} v s .18 \mathrm{~d})$. The good agreement also applies to three additional chemicals (acesulfame, carbamazepine, and fluconazole) that were relatively persistent in both studies.

\subsection{Chemical persistence in the laboratory test}

There were many chemicals quantifiable in the laboratory test that were not quantified in the field. This was expected because 
Table 1 Summary of chemicals with a degradation half-life of $<100 \mathrm{~d}$ in the field

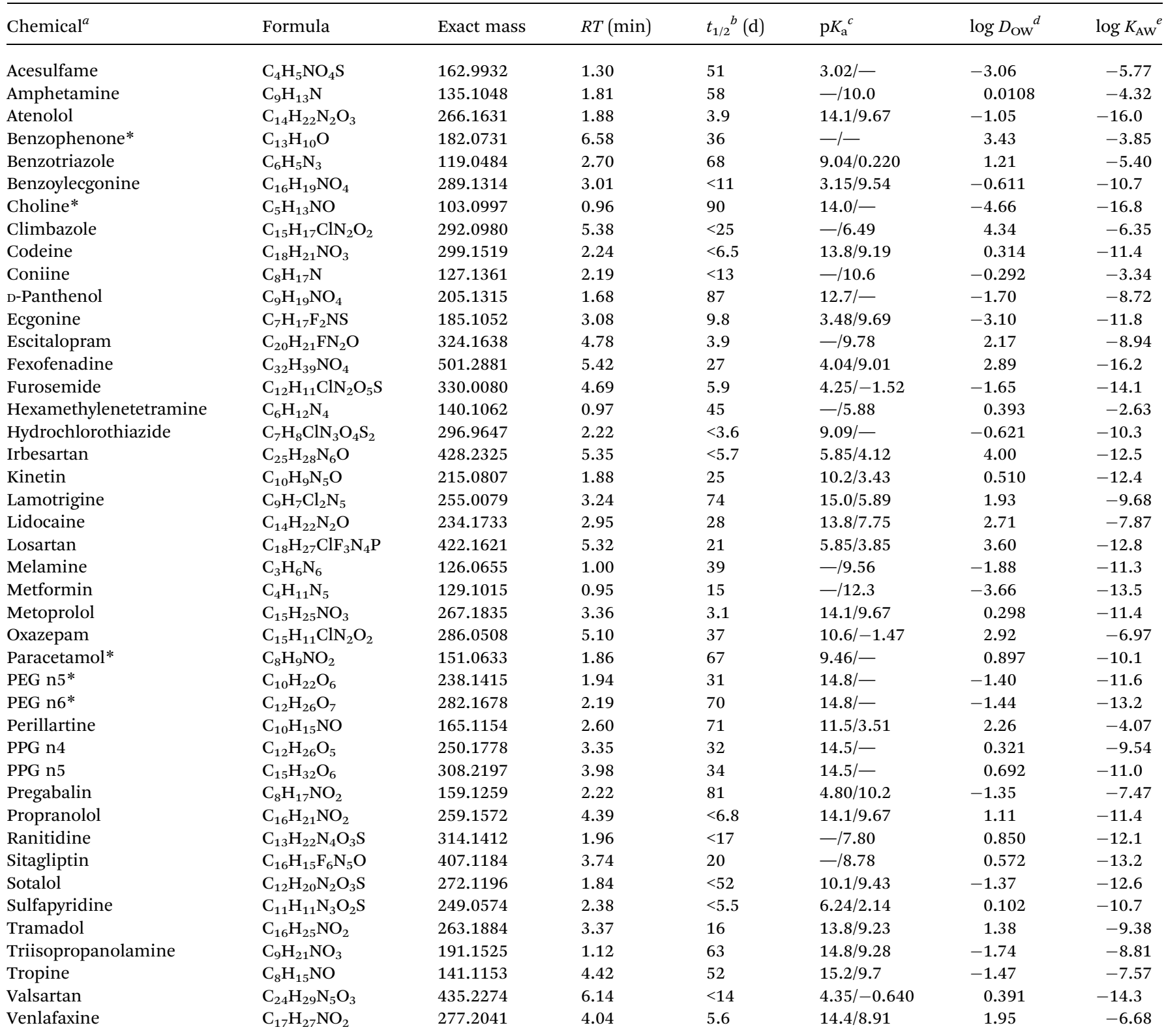

${ }^{a}$ Chemicals with asterisks indicate an inflow contribution from Södra Bergundasjön of $>30 \% .{ }^{b}$ The sign $<$ is used before $t_{1 / 2}$ if the concentration was $<$ LOD in outflow, in which case the calculated $t_{1 / 2}$ represents a maximum value. ${ }^{c} \mathrm{p} K_{\mathrm{a}}$ values were obtained from ChemAxon; numbers before and after the slash indicate the strongest acidic and basic $\mathrm{p} K_{\mathrm{a}}$ values, respectively. ${ }^{d} \log D_{\mathrm{OW}}$ values were calculated using the $\log K_{\mathrm{OW}}$ value of a chemical at the measured ambient $\mathrm{pH}$ value of 8.2 in the unspiked bottles; $\log K_{\mathrm{OW}}$ values were obtained from ChemAxon. ${ }^{e} \log K_{\mathrm{AW}}$ values were calculated from $\log K_{\mathrm{OW}}$ and $\log K_{\mathrm{OA}}$ that was obtained from EPI Suite.

we chose to study a lake with a long water residence time so that it would be possible measure half-lives in the field that were close to the regulatory thresholds for persistence. The long water residence time meant that chemicals with short half-lives, some of which could be measured in the laboratory test, were not detectable in the field.

Out of the 38 chemicals that were detected in the field with a half-life of $<100 \mathrm{~d}$ and for which the WWTP effluent was the dominant source to the lake, half-lives could be determined for 32 in the bottle test with mixture lake water. This was possible for only 20 of the 38 in the bottles with natural water (half-lives are provided in Table $\mathrm{S} 4 \dagger$ ). For the 20 chemicals quantified in both bottle tests, there was no significant difference between the half-life in the mixture lake water and the half-life in the natural water for 17 chemicals, the exceptions being perillartine, PPG n4, and PPG n5. The half-lives of PPG n4 and PPG $\mathrm{n} 5$ in the mixture lake water were shorter than those in the natural water by a factor of 4 and 7 , respectively. Perillartine was relatively persistent in the natural water with the $95 \%$ confidence interval of its estimated half-life intersecting infinity (Table S4 $\dagger$ ). The faster dissipation of these 3 chemicals in the mixture lake water might be due to more specialized degrading 
capacity of the microbial population in the effluent compared to the lake water, so that even though effluent only made up $20 \%$ of the test medium it still resulted in faster biodegradation. Based on the good agreement between mixture lake water and natural water for $85 \%$ of the chemicals, the half-lives from the bottle tests with mixture lake water were used for comparison with the field measurements.

Of the 32 chemicals quantified in the bottle tests with mixture lake water, 18 were degraded in the lab test following first-order kinetics (Fig. 2). For one of these, sulfapyridine, concentrations increased again after $10 \mathrm{~d}$, suggesting that the initial transformation may have been reversible or that it was formed as the product of the transformation of another compound. For the remaining 14 chemicals, four had poor measurement repeatability so that the uncertainty in half-life was large, while the other ten were either very persistent with half-life confidence intervals intersecting infinity or they were formed from precursor chemicals. Of the 18 chemicals clearly showing first-order dissipation, the linear regression of $\ln$ (concentration) versus time yielded a coefficient of determination $\left(R^{2}\right)>0.90$, and a lag phase was observed for only three: acesulfame, furosemide, and losartan. One advantage of the unspiked bottle test over the traditional spiked test is that the level of substrate is not modified. Spiking can result in adaptation of the microflora, leading to both a lag phase and potentially a different elimination rate than in the environment. ${ }^{6}$ Although we did not spike in our experiment, the mixing of surface water and effluent in this experiment created new contaminant levels for the microorganisms present. This could explain the lag phase observed for furosemide and losartan. Acesulfame is degraded by a specialized organism in WWTPs, and this organism may have been present in sufficient quantities in the WWTP effluent and found suitable conditions in the mixture lake water incubation for growth, leading to the observed lag. ${ }^{18}$ No statistically significant difference in half-life between the two replicate bottles was observed for any of the 18 chemicals (Fig. 2), indicating good repeatability of the half-life determination. For comparison with the field measurements the data from the two bottles were combined (Table S4 $\dagger$ ).

\subsection{Comparison of persistence in the field and the laboratory}

Field and lab persistence calculated for the 32 chemicals that were quantifiable in both experiments are plotted as Fig. 3 and summarized in Table S4. $\uparrow$ The discussion below is divided into two parts based on: (i) the 18 chemicals for which the degradation in the lab test followed first-order kinetics and the halflife was determined with high confidence, and (ii) the remaining 14 chemicals for which the half-life in the lab test had large uncertainty or the $95 \%$ confidence intervals intersected infinity.

3.6.1. Chemicals for which half-life was calculated with high confidence. Of the 18 chemicals for which degradation followed first-order kinetics (the left part of Fig. 3), good

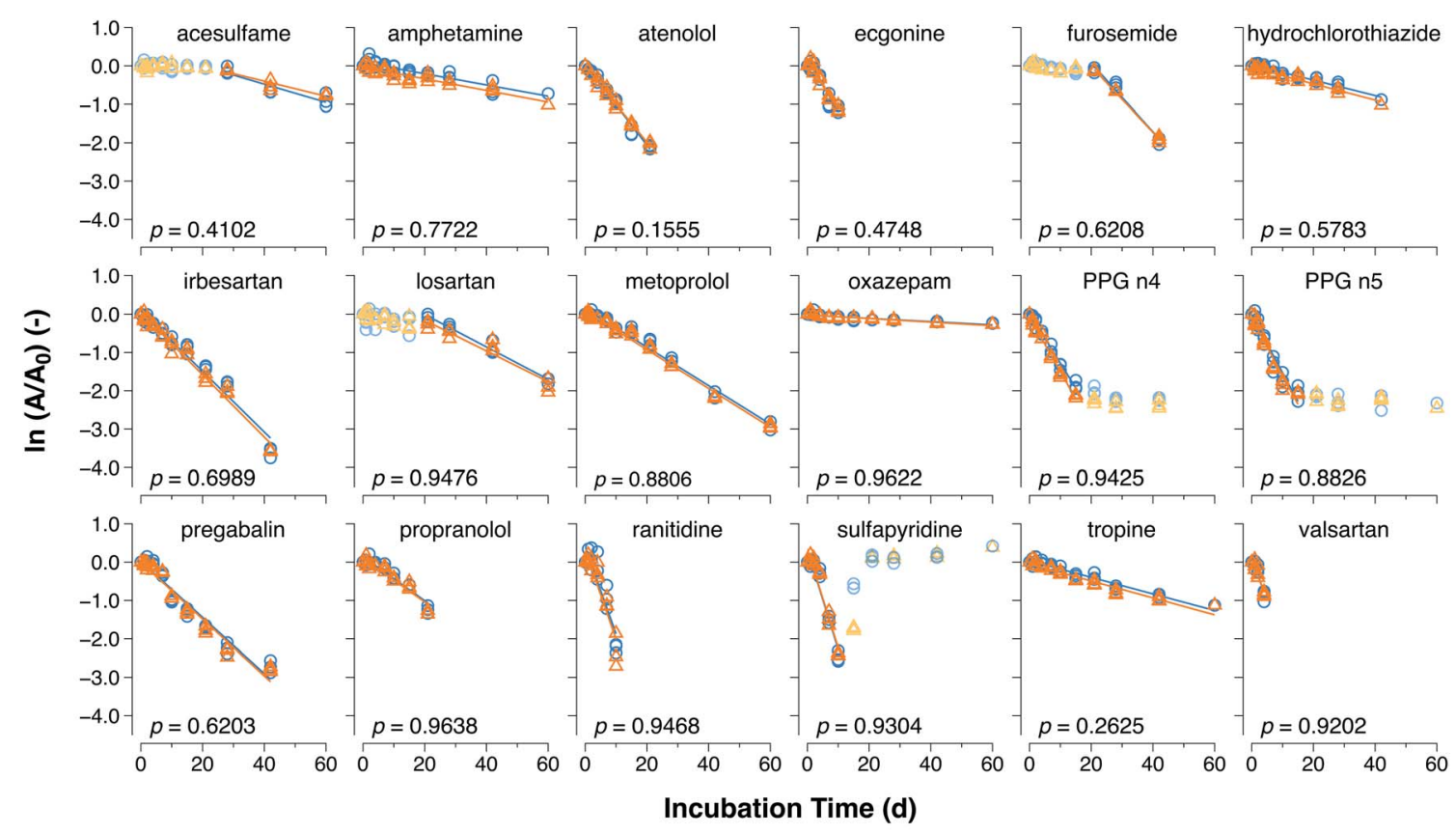

- bottle 1

$\triangle \quad$ bottle 2

linear regression for bottle 1

linear regression for bottle 2

Fig. 2 Dissipation of the 18 chemicals showing $1^{\text {st }}$ order kinetics in the unspiked OECD 309 tests. The results for two replicate experiments are shown, bottle 1 in blue and bottle 2 in orange. All graphs share the same $x$ and $y$ axis and show the peak area normalized to the initial peak area. Linear regressions are shown as solid lines. The symbols in lighter colors show data points that were not used in determining the linear regressions because they were indicative of a lag phase or a sudden decrease in dissipation rate. The $p$ value in each graph indicates the ANCOVA test result comparing the two regression lines; no significant difference was observed for any chemical at the $95 \%$ confidence level $(p>0.05)$. 


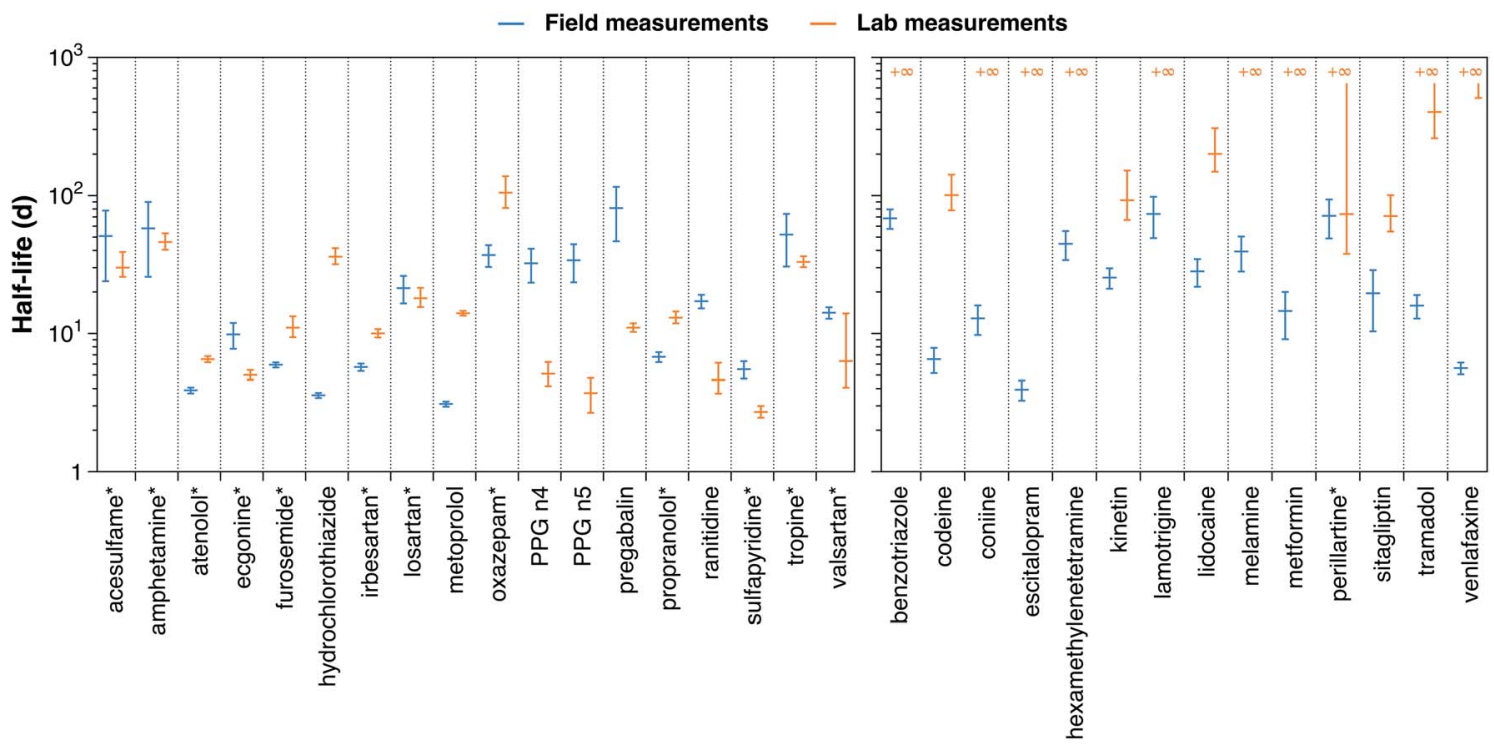

Fig. 3 Comparison of half-lives measured in the lab experiment with mixture lake water and in the field. The $*$ symbol next to a chemical indicates a good agreement between field and lab half-lives (central estimates differ by a factor of $<3$ ). The $+\infty$ symbol indicates that the slope of the linear regression was either positive or not significantly different from zero $(p>0.05)$. The error bars show the standard deviation for the field measurements and the $95 \%$ confidence interval for the lab measurements.

agreement between the field half-life and lab half-life measured in mixture lake water was found for 12 substances (indicated with asterisks in Fig. 3; defined as a difference of less than a factor three in the central estimates of the half-lives (illustrated in Fig. S6 $†$ )). Four of the other six chemicals (PPG n4, PPG $\mathrm{n} 5$, pregabalin, and ranitidine) had a markedly longer half-life in the field than in the lab test. The lab experiment may have shown a faster dissipation because it was done with an effluent/ lake water mixture, which may have had a microbial population more adapted to degrading these substances. Indeed, when the lab test half-lives of PPG n4 and PPG n5 measured in natural water where compared with the field half-lives, there was good agreement (Table S4 $\dagger$ ). The half-life of pregabalin could not be measured in the lab test with natural water, so we cannot judge whether the same explanation also applies to these two chemicals. The field half-life for ranitidine had a lower confidence interval of zero (the concentrations in lake water were below the LOQ), and therefore the difference between the field and lab half-lives was not significant.

In the case of the other two chemicals, hydrochlorothiazide and metoprolol, the half-lives measured in the field were 10 and 4 times shorter, respectively, than the half-lives measured in the lab (Table S4†). Although it has been documented that dissipation of hydrochlorothiazide in lab degradation tests can be attributed to both biodegradation and non-photolytic abiotic transformation (e.g., hydrolysis) ${ }^{19,20}$ neither biodegradation nor hydrolysis is likely to explain the substantially faster dissipation of hydrochlorothiazide observed in the field. Photodegradation of hydrochlorothiazide has also been reported and was found to be a dominant dissipation pathway under certain conditions. ${ }^{21-23}$ Given the generally high global radiation and long sunshine hours during the sampling period (Fig. S5†), we hypothesize that hydrochlorothiazide was removed from Lake
Norra Bergundasjön primarily via photodegradation. Such conclusions are also consistent with Zou et al. (2015b), who studied seasonal variation of the persistence of 12 chemicals including hydrochlorothiazide in another Swedish lake. ${ }^{12}$ In that study, the largest seasonal difference was observed for hydrochlorothiazide, for which the calculated half-life in late spring (with high irradiance) was 20 times lower than in late autumn (with low irradiance). The correlation between removal rate and irradiance suggests photodegradation as the dominant removal process, while a seasonality in chemical persistence of this magnitude is unlikely for other degradation pathways such as biodegradation or hydrolysis. Although Lake Norra Bergundasjön is a hypertrophic lake with strong algal blooms during spring and summer which would limit light penetration, the relatively shallow depth of the lake favors photochemical removal processes.

Other transformation processes may also explain the shorter half-life of metoprolol in the field. Metoprolol is subject to indirect photodegradation in the presence of natural organic matter. ${ }^{24}$ It has also been shown to degrade rapidly at warm temperatures $\left(19.7^{\circ} \mathrm{C}\right)$ under oxic/penoxic to suboxic conditions in sediment. ${ }^{25}$

3.6.2. Chemicals for which half-life was calculated with low confidence or intersected infinity. The time trends of the 14 chemicals for which the half-life obtained in the lab was uncertain or its $95 \%$ confidence interval intersected infinity are plotted as Fig. S7. $\dagger$ Ten of these chemicals (indicated with $+\infty$ in Fig. 3) were either very persistent in the lab or were formed during the 60 day incubation period (Fig. S7†). For the remaining four chemicals the half-life was so uncertain that comparison with the field half-life does not provide much useful information about the ability of the lab test to predict biodegradation in the field (Fig. S7†). The cause of the high 
uncertainty was large variability in the measured concentrations, which may suggest that the analytical approach used was not suitable for these chemicals.

The half-life in the field for these chemicals covers a wide range, from $3.9 \mathrm{~d}$ (for escitalopram) to $74 \mathrm{~d}$ (for lamotrigine) (see the right part of Fig. 3 and Table $S 4 \dagger$ ). All of the 14 chemicals except perillartine had a half-life in the lab significantly longer than in the field. Analogous to hydrochlorothiazide, photodegradation may explain the dissipation of some of these chemicals in Lake Norra Bergundasjön. For instance, venlafaxine has been shown to be degraded via indirect photolytic reactions. ${ }^{26}$ Another possible explanation is that a major portion of the biodegradation of these chemicals in the field is occurring in the sediment. In flowing systems the hyporheic exchange occurring at the water/sediment interface has been demonstrated to play a significant role in biodegradation of organic contaminants. ${ }^{27-29}$ Further work is needed to explore these hypotheses. However, this was beyond the scope of this study.

\subsection{Potential and limitations of the unspiked OECD 309 test}

The unspiked OECD 309 test was a good predictor of dissipation half-lives in the field. Of the 18 chemicals for which half-lives could be quantified with reasonable certainty in the lab test, 14 chemicals showed satisfactory agreement with the field halflives. This shows that the unspiked OECD test has potential for assessing dissipation half-lives of contaminants in specific field situations.

However, the experiments also revealed some of the limitations of this methodology. The dissipation kinetics of some chemicals could not be measured in the laboratory test with natural water because the concentrations were too low. Using the mixture lake water instead, more chemicals could be analyzed and the confidence intervals were narrowed for other chemicals that had been quantified in the natural water. This indicates that mixture lake water is a feasible strategy for overcoming the limitation of low contaminant concentrations in natural water. However, although there was good agreement between the half-lives measured in natural water and mixture lake water for most chemicals, several disagreed substantially. This indicates that the uncertainties with respect to microbial composition associated with this strategy can be substantial, and that it should be used with caution. Improving the sensitivity of analytical methods is ultimately a more attractive solution to the concentration limitation of the unspiked OECD 309 test.

A second limitation of the laboratory test is that it does not account for all loss mechanisms. Since only water is studied, the laboratory test cannot account for attenuation occurring in sediments, and there is ample evidence showing that sediments possess a strong biodegradation capacity for some chemicals. The OECD 309 test has the option of adding sediment to the incubation, but there is no guidance on how much sediment should be added so that the balance between biotransformation capacity in sediment and water is the same in the lab test as it is in the field. Furthermore, the unspiked OECD 309 test does not consider photodegradation. This could be addressed by conducting separate photodegradation experiments in the laboratory and then using theoretical considerations to translate the results into field persistence. ${ }^{30,31}$

Finally, the unspiked laboratory test is not well suited for the identification of transformation products. In a spiked test the identification of transformation products is fostered by the perturbation of the system; transformation products can be identified by searching for chemicals which show an increase in concentration. This is not possible in the unspiked test. However, once transformation products have been identified, the unspiked test offers good opportunities to study their occurrence and relevance under natural conditions. This is because the complex mixture of potential reactants found in the environment that could influence the concentration of transformation products is reproduced in the laboratory test.

\section{Conflicts of interest}

There are no conflicts to declare.

\section{Acknowledgements}

The authors thank Anneli Andersson Chan and her colleagues at the Sundet Wastewater Treatment Plant for their assistance in obtaining the effluent and lake water and Andreas Hedrén from Växjö Municipality for sharing the flow rate data. The Swedish Research Council FORMAS is acknowledged for funding this study.

\section{References}

1 European Chemical Agency ECHA, Guidance on Information Requirements and Chemical Safety Assessment Chapter R.11: $P B T / v P v B$ assessment, Helsinki, Finland, 2017, pp. 1-158.

$2 \mathrm{M}$. Scheringer, Persistence and spatial range as endpoints of an exposure-based assessment of organic chemicals (vol 30, pg 1652, 1996), Environ. Sci. Technol., 1996, 30(8), 2660.

3 J. A. Arnot, T. N. Brown, F. Wania, K. Breivik and M. S. McLachlan, Prioritizing chemicals and data requirements for screening-level exposure and risk assessment, Environ. Health Perspect., 2012, 120(11), 15651570.

$4 \mathrm{M}$. Honti and K. Fenner, Deriving persistence indicators from regulatory water-sediment studies - opportunities and limitations in OECD 308 data, Environ. Sci. Technol., 2015, 49(10), 5879-5886.

5 Organisation for Economic Co-operation and Development OECD, OECD Guidelines for the Testing of Chemicals 309, Paris, 2004, pp. 1-21.

6 Z. Li and M. S. McLachlan, Biodegradation of chemicals in unspiked surface waters downstream of wastewater treatment plants, Environ. Sci. Technol., 2019, 53(4), 18841892.

7 B. A. Kolvenbach, D. E. Helbling, H.-P. E. Kohler and P. F.-X. Corvini, Emerging chemicals and the evolution of 
biodegradation capacities and pathways in bacteria, Curr. Opin. Biotechnol., 2014, 27, 8-14.

8 A. Kowalczyk, T. J. Martin, O. R. Price, J. R. Snape, R. A. van Egmond, C. J. Finnegan, H. Schäfer, R. J. Davenport and G. D. Bending, Refinement of biodegradation tests methodologies and the proposed utility of new microbial ecology techniques, Ecotoxicol. Environ. Saf., 2015, 111, 9-22.

9 H. Zou, M. Radke, A. Kierkegaard, M. MacLeod and M. S. McLachlan, Using chemical benchmarking to determine the persistence of chemicals in a Swedish lake, Environ. Sci. Technol., 2015, 49(3), 1646-1653.

10 Z. Li, E. Undeman, E. Papa and M. S. McLachlan, Highthroughput evaluation of organic contaminant removal efficiency in a wastewater treatment plant using direct injection UHPLC-Orbitrap-MS/MS, Environ. Sci.: Processes Impacts, 2018, 20, 561-571.

11 E. L. Schymanski, J. Jeon, R. Gulde, K. Fenner, M. Ruff, H. P. Singer and J. Hollender, Identifying small molecules via high resolution mass spectrometry: communicating confidence, Environ. Sci. Technol., 2014, 48(4), 2097-2098.

12 H. Zou, M. Radke, A. Kierkegaard and M. S. McLachlan, Temporal variation of chemical persistence in a Swedish lake assessed by benchmarking, Environ. Sci. Technol., 2015, 49(16), 9881-9888.

13 R. H. Lindberg, P. Wennberg, M. I. Johansson, M. Tysklind and B. A. V. Andersson, Screening of human antibiotic substances and determination of weekly mass flows in five sewage treatment plants in Sweden, Environ. Sci. Technol., 2005, 39(10), 3421-3429.

14 R. H. Lindberg, J. Fick and M. Tysklind, Screening of antimycotics in Swedish sewage treatment plants-waters and sludge, Water Res., 2010, 44(2), 649-657.

15 M. Östman, J. Fick, E. Näsström and R. H. Lindberg, A snapshot of illicit drug use in Sweden acquired through sewage water analysis, Sci. Total Environ., 2014, 472, 862871.

16 M. Östman, R. H. Lindberg, J. Fick, E. Björn and M. Tysklind, Screening of biocides, metals and antibiotics in Swedish sewage sludge and wastewater, Water Res., 2017, 115, 318328.

17 Z. Li, S. L. Kaserzon, M. M. Plassmann, A. Sobek, M. J. Gómez Ramos and M. Radke, A strategic screening approach to identify transformation products of organic micropollutants formed in natural waters, Environ. Sci.: Processes Impacts, 2017, 19, 488-498.

18 S. Kahl, S. Kleinsteuber, J. Nivala, M. van Afferden and T. Reemtsma, Emerging biodegradation of the previously persistent artificial sweetener acesulfame in biological wastewater treatment, Environ. Sci. Technol., 2018, 52(5), 2717-2725.

19 Z. Li, M. P. Maier and M. Radke, Screening for pharmaceutical transformation products formed in river sediment by combining ultrahigh performance liquid chromatography/high resolution mass spectrometry with a rapid data-processing method, Anal. Chim. Acta, 2014, 810, 61-70.

20 M. Radke and M. P. Maier, Lessons learned from water/ sediment-testing of pharmaceuticals, Water Res., 2014, 55, 63-73.

21 A. Jaeger, M. Posselt, A. Betterle, J. Schaper, J. Mechelke, C. Coll and J. Lewandowski, Spatial and temporal variability in attenuation of polar organic micropollutants in an urban lowland stream, Environ. Sci. Technol., 2019, 53(5), 2383-2395.

22 A. L. Boreen, W. A. Arnold and K. McNeill, Photodegradation of pharmaceuticals in the aquatic environment: a review, Aquat. Sci., 2003, 65(4), 320-341.

23 M. Brigante, M. DellaGreca, L. Previtera, M. Rubino and F. Temussi, Degradation of hydrochlorothiazide in water, Environ. Chem. Lett., 2005, 2(4), 195-198.

24 O. M. S. Filipe, N. Mota, S. A. O. Santos, M. R. M. Domingues, A. J. D. Silvestre, M. G. P. M. S. Neves, M. M. Q. Simões and E. B. H. Santos, Identification and characterization of photodegradation products of metoprolol in the presence of natural fulvic acid by HPLC-UV-MS ${ }^{n}, J$. Hazard. Mater., 2017, 323, 250-263.

25 V. Burke, J. Greskowiak, T. Asmuß, R. Bremermann, T. Taute and G. Massmann, Temperature dependent redox zonation and attenuation of wastewater-derived organic micropollutants in the hyporheic zone, Sci. Total Environ., 2014, 482-483, 53-61.

26 P. C. Rúa-Gómez and W. Püttmann, Degradation of lidocaine, tramadol, venlafaxine and the metabolites $\mathrm{O}$ desmethyltramadol and O-desmethylvenlafaxine in surface waters, Chemosphere, 2013, 90(6), 1952-1959.

27 A. Jaeger, C. Coll, M. Posselt, J. Mechelke, C. Rutere, A. Betterle, M. Raza, A. Mehrtens, K. Meinikmann, A. Portmann, et al., Using recirculating flumes and a response surface model to investigate the role of hyporheic exchange and bacterial diversity on micropollutant half-lives, Environ. Sci.: Processes Impacts, 2019, 21(12), 2093-2108.

28 Z. Li, A. Sobek and M. Radke, Flume experiments to investigate the environmental fate of pharmaceuticals and their transformation products in streams, Environ. Sci. Technol., 2015, 49(10), 6009-6017.

29 J. L. Schaper, M. Posselt, J. L. McCallum, E. W. Banks, A. Hoehne, K. Meinikmann, M. A. Shanafield, O. Batelaan and J. Lewandowski, Hyporheic exchange controls fate of trace organic compounds in an urban stream, Environ. Sci. Technol., 2018, 52(21), 12285-12294.

30 C. Prasse, J. Wenk, J. T. Jasper, T. A. Ternes and D. L. Sedlak, Co-occurrence of photochemical and microbiological transformation processes in open-water unit process wetlands, Environ. Sci. Technol., 2015, 49(24), 14136-14145.

31 R. M. Baena-Nogueras, E. González-Mazo and P. A. LaraMartín, Degradation kinetics of pharmaceuticals and personal care products in surface waters: photolysis $v s$. biodegradation, Sci. Total Environ., 2017, 590-591, 643-654. 